\title{
MIGRACIÓN INTERNA, MOVILIDAD SOCIAL Y ACTITUDES Y ORIENTACIONES DE TRABAJADORES PERUANOS
}

\author{
Martín J. Scurrah \\ AbNer Montalvo V. \\ Escuela de Administración para Graduados de Lima
}

\section{INTRODUCCIÓN}

UNo DE LOS CAMBIos más profundos experimentado por la sociedad peruana en los años posteriores a la segunda guerra mundial, ha sido el de la migración interna masiva, mayormente de la Sierra a la Costa y en especial a Lima, pero también de la Sierra a la Selva y de ésta a Lima (Centro de Estudios de Población y Desarrollo, 1972: 163; Oficina Nacional de Estadística y Censos, 1972: 90)..$^{1}$

Los estudios del proceso migratorio en el Perú (véase, por ejemplo, el recuento que hacen Alers y Appelbaum, 1968) son mayormente antropológicos. Algunos de ellos se han basado en el análisis de los datos de los censos (véase, por ejemplo, Martínez, 1968) y otros en información proveniente de encuestas (véase Valdivia Ponce, 1970). Sin embargo, hay mucho campo para estudios que permitan examinar variables del proceso de migración usando instrumentos de análisis más sofisticados que los que se han considerado hasta ahora.

El presente trabajo intenta analizar el impacto del proceso de migración en las actitudes y valores, la adaptación psicológica y la movilidad ocupacional y social de los trabajadores en el sector comercial de Lima. Se sabe que la industria y el comercio de Lima ocupan una elevada proporción de trabajadores nacidos o criados fuera de Lima. También se sabe que hay diferencias culturales muy importantes entre Lima y el resto del Perú, especialmente en relación con la Sierra y la Selva. Pero se conoce muy poco acerca de la influencia de estas diferencias culturales en las actitudes y el comportamiento en los centros de trabajo. ¿Muestran actitudes y valores diferentes los trabajadores nacidos o socializados o ambas cosas fuera de Lima o quienes han vivido la mayor parte de su vida fuera de Lima, de las que muestran los trabajadores

1 Los autores agradecen al Prof. Hutgo Serrano por su valiosa ayuda en la supervisión de la encuesta, y al Dr. Fred Zappert y a la Oficina de Informática del Ministerio de Economía y Finanzas por su ayuda en el procesamiento de datos. 
nacidos o criados en Lima o aquellos que han vivido la mayor parte de su vida en esa ciudad?; ¿los trabajadores inmigrantes muestran mayor incidencia de problemas psicológicos, debido a las dificultades encontradas para su adaptación a la ciudad, en relación con los trabajadores nativos; o muestran menos problemas, debido a su menor exposición a las presiones psicológicas del ambiente urbano?; ¿experimentan los trabajadores inmigrantes un proceso de movilidad social ascendente, o simplemente reemplazan a los trabajadores urbanos en los cuadros más bajos de la estructura económico-social de la ciudad? Si existen diferencias entre los trabajadores nativos y los trabajadores inmigrantes ise deben éstas a las diferencias culturales entre la ciudad y el campo; o a las diferencias entre las diversas áreas ecológico-culturales del país; o simplemente se deben a las diferencias de exposición al ambiente metropolitano de Lima?

El presente análisis se basa en una encuesta llevada a cabo en 1972 en tres empresas comerciales de Lima Metropolitana. Cada empresa consta de más de una tienda, de tal manera que el total de unidades organizacionales (tiendas y almacenes) incluidas en la encuesta, es de 22 . La muestra de trabajadores fue estratificada por división organizacional y por nivel jerárquico. El cuestionario se aplicó a un total de 792 trabajadores: $63 \%$ hombres y $37 \%$ mujeres; $46 \%$ nacieron en Lima o Callao y $54 \%$ nacieron fuera de estas ciudades.

\section{VARIABLES INDEPENDIENTES}

Al utilizar como guía las categorías desarrolladas por Browning y Feindt (1968) en su análisis de la migración interna en México, se confeccionó un continuo de exposición al ambiente urbano. Este continuo consta de las siguientes cinco categorías:

1) nativos nacidos en Lima-Callao;

2) nativos por adopción (nacidos fuera de la ciudad pero cuyos años de formación transcurrieron en ella);

3) migrantes con un período largo de exposición (no nacidos en la ciudad);

4) migrantes con un período intermedio de exposición (no nacidos ni formados en la ciudad pero que han vivido en ella de 10 a 9 años); y

5) migrantes con un período corto de exposición (no nacidos ni formados en la ciudad, y con menos de 10 años de residencia en ella).

Este continuo resume la información de varios elementos del proceso de migración; así pues, nos parece conveniente analizar también otras variables independientes tales como: 1) lugar de nacimiento; 2) lugar de socialización; 3) lugar donde pasó la mayor parte de su vida. Cada una 
de estas variables se considera en términos de dos dimensiones: 1) dimensión rural-urbana con las categorías Lima-Callao, capital de departamento, capital de provincia y distrito, pueblo o campo; 2) dimensión ecológico-cultural con las categorías Lima-Callao, Costa fuera de Lima, Selva y Sierra. Se podría objetar que el uso de capitales de departamentos, de provincias o de distritos constituye sólo una medida aproximada de su tamaño y grado de urbanización, pero, en general, éstas forman un continuo y, dadas las limitaciones de un cuestionario y la falta de cifras exactas y actualizadas, no ha quedado otra alternativa que la de recurrir a esta referencia. La dimensión ecológico-cultural se construyó con base en el grado de similitud cultural con Lima-Callao. Se calificó la Selva como más parecida a Lima-Callao que la Sierra, porque los migrantes de la Selva a Lima son mestizos casi en su totalidad - no provenientes de tribus nativas- y porque otros estudios (por ejemplo, Valdivia Ponce, 1970) indican que este orden es el apropiado.

\section{VARIABLES DEPENDIENTES}

Se ha considerado conveniente analizar tres grupos de variables dependientes. El primero comprende las actitudes y orientaciones de los trabajadores encuestados. Por eso se ha establecido una escala de optimismo y subescalas de optimismo en comparación con el pasado, optimismo acerca del futuro, optimismo acerca del país y optimismo acerca de 1a situación personal del entrevistado. También hemos establecido una escala general de fatalismo y subescalas de fatalismo acerca de la utilidad de trabajar duro, fatalismo acerca del valor de una buena preparación personal, fatalismo acerca de la suerte y fatalismo acerca de "vara". La confianza se ha medido mediante una escala breve y otra mayor y más completa. Se ha considerado la alienación en forma general y también utilizando subescalas que miden la alienación del puesto, la alienación de la empresa y la alienación del grupo de trabajo. Además de estas actitudes importantes se han tomado en cuenta algunas orientaciones generales tales como la orientación en el tiempo (sea hacia el pasado o hacia el futuro); el grado de autoritarismo del individuo (una característica de su personalidad) y su preferencia por el valor de independencia frente al conformismo. Finalmente, se ha establecido una escala para medir la solidaridad del grupo en que trabajaba el entrevistado. ${ }^{2}$

2 El optimismo en comparación con el pasado se midió por una escala que comprende los siguientes items: si en los últimos cinco años los ricos se han hecho más ricos y los pobres más pobres; si la situación general del país ha mejorado o empeorado en los últimos cinco años; si la situación personal del entrevistado ha mejorado o empeorado en los últimos cinco años; y, en general, cómo compara el presente con el pasado. El optimismo acerca del futuro se midió por una escala que incluye los siguientes ítems: si la situación del país mejorará o empeorará en los próximos cinco años; si la situación personal del entrevistado mejorará o empeorará en los próximos cinco años; si el ingreso del entrevistado aumentará o 
El segundo grupo de variables dependientes comprende: satisfacción con las aspiraciones alcanzadas; nivel de las aspiraciones para el futuro, expresadas por medio de los hijos; satisfacción general respecto al tra-

disminuirá en los próximos cinco años; y, en general, cómo compara el presente con el futuro. Se midió el optimismo acerca del país por medio de una escala que comprende los siguientes items: si los ricos se habían hecho más ricos y los pobres más pobres en los últimos cinco años; si la situación general del país ha mejorado o empeorado en los últimos cinco años; y si la situación general del país mejorará o empeorará en los próximos cinco años. El optimismo del entrevistado acerca de su situación personal se calculó por una escala que incluye los siguientes items: si la situación personal del entrevistado ha mejorado o empeorado en los últimos cinco años; si su situación personal mejorará o empeorará en los próximos cinco años; comparación del presente con el pasado; y comparación del presente con el futuro. Finalmente, se desarrolló una escala total de optimismo de los siguientes índices: si los ricos se habían hecho más ricos y los pobres más pobres; la situación general del país en comparación con el pasado y con el futuro; la situación personal del entrevistado en comparación con el pasado y con el futuro; la predicción del entrevistado acerca de sus futuros ingresos; y el presente comparado con el pasado y con el futuro.

El fatalismo acerca de la utilidad de trabajar duro se midió por una escala que responde a las siguientes preguntas:

Para progresar en la vida, Zes más importante tener buena suerte que ser trabajador?; para progresar en la vida, ¿es más importante tener "vara" o influencia que ser trabajador? Se midió el fatalismo en contraste con la preparación personal, mediante una escala que comprende los dos siguientes índices: para triunfar en la vida, ¿es más importante tener preparación personal que "vara" o influencia?; el éxito en la vida idepende más de la suerte que de la preparación personal? Se calcularon dos escalas más empleando diferentes combinaciones de los siguentes cuatro planteamientos: fatalismo en términos de suerte, utilizando las preguntas que contrastan la suerte con el trabajo duro y con la preparación personal, y fatalismo en términos de "vara" o influencia, empleando las preguntas que contrastan la influencia con el trabajo duro y con la preparación personal. Finalmente, se estableció una escala total de fatalismo incluyendo los cuatro índices ya mencionados más las siguientes preguntas: ¿puede cambiar su propio destino?; ¿algunos han nacido para mandar y otros para obedecer?; ies mejor disfrutar lo que hoy nos brinda la vida que esperar el futuro?

Se desarrollaron dos escalas para medir la confianza. La escala más completa comprende lo siguiente: ¿hoy en día no se sabe en quién confiar?; si uno no se cuida a sí mismo, ¿la gente se aprovechará?; ¿puede confiar en la mayoría de la gente?; si usted fracasa, ¿a nadie le importará?; cuando uno se encuentra en dificultades, ¿la gente acude espontáneamente a ayudarlo?; ¿no conviene que los compañeros de labor se enteren de todo lo que uno sabe acerca del trabajo porque pueden aprovecharse?; icree que hoy se puede confiar mucho, regularmente o no se puede confiar en: parientes, amigos, vecinos, y el resto de la gente? Además se calculó una versión más breve de la escala empleando los cuatro índices de la última pregunta de la escala general.

Se estableció una escala total y tres subescalas de alienación. La alienación del puesto incluye mediciones del aburrimiento, de la sensación del tiempo, de las oportunidades para aprender cosas nuevas, del futuro que deparará el puesto y de las oportunidades para usar las habilidades en el trabajo. La alienación del grupo de trabajo se estableció midiendo el grado de amistad con los compañeros de trabajo y su voluntad de ayudar en caso de una desgracia; y la alienación a la empresa, midiendo su percepción respecto a la preocupación de dicha empresa por cada trabajador, si la empresa trata igualmente a los trabajadores en idénticas 
bajo y la empresa; el deseo de mejorar; la movilidad social intergeneracional y la movilidad ocupacional. ${ }^{3}$

El tercer grupo de variables dependientes se refiere a dos medidas de la adaptación psicológica del individuo. Se incluyeron en el cuestionario

circunstancias y por la percepción que el trabajador tiene acerca de su habilidad para influir en las condiciones de su trabajo. La escala total de alienación incluye todos estos índices más los de satisfacción con el puesto, tratamiento recibido de los funcionarios públicos y percepción de la situación general por la mayoría de la gente.

Se calculó una escala de la orientación temporal del entrevistado con base en las siguientes indicaciones: si es más importante el pasado, el presente 0 el futuro; si todo tiempo pasado fue mejor; el presente comparado con el pasado; el presente comparado con el futuro; si es mejor aprovechar lo que nos brinda hoy la vida que esperar el futuro; y definición del entrevistado respecto al "futuro".

Se han usado los siguientes índices para establecer una escala estimando el valor que se da a la independencia versus el conformismo: la habilidad percibida para controlar el destino, si algunos nacen para mandar y otros para obedecer, si se quiere más o menos control ejercido por el supervisor y los alcances del control deseado.

Se determinó el grado de autoritarismo del entrevistado combinando las respuestas a una pregunta sobre el grado de control del jefe y a otra sobre el grado de control deseado. Se calificaron como autoritarios aquellos que estaban experimentando mucho control y que deseaban igual o más control; y como antiautoritarios aquellos que estaban experimentando poco control y que deseaban igual grado de control o menos.

Se midió la solidaridad del grupo de trabajo por una escala compuesta de los siguientes índices: si trabajando más uno será objeto de envidia o admiración; si trabajar más se considerará positivo o negativo para el grupo; si trabajar más deparará simpatía o antipatía; si trabajando más el grupo le ayudaría o le obstaculizaría; satisfacción con los compañeros de trabajo; amistad de los compañeros de trabajo; si los compañeros de trabajo se llevan bien; la ayuda mutua de los compañeros de trabajo; si está dispuesto a cambiar de grupo; y la comparación de su grupo con otros en la empresa.

3 Se estableció una escala midiendo las aspiraciones alcanzadas a partir de la satisfacción con el logro alcanzado en el trabajo; de la satisfacción de los padres por los logros de uno en su trabajo; y de la comparación del éxito de uno con el logrado por los parientes y amigos. Las aspiraciones para el futuro se midieron mediante preguntas sobre el prestigio de la ocupación preferida para su hijo y para su hija. Se midió la satisfacción en el trabajo por una escala de tres items: satisfacción con los ingresos; satisfacción con los compañeros de trabajo; y si le gusta su trabajo.

Se estimó el deseo de mejorar del entrevistado mediante una escala que combina dos planteamientos: si resulta o no trabajar hasta agotarse pues nadie se lo reconoce a uno, y si se iría de su puesto actual sin vacilar, o sólo después de pensarlo mucho, o no se iría si tuviera una oportunidad de mejorar su nivel de vida en otra empresa.

Se calculó la movilidad social intergeneracional juntando en una escala la clase social del entrevistado comparada con la de su padre; de su educación comparada con la de su padre y de su educación comparada con la de su madre. La movilidad ocupacional se midió combinando en una escala la medida del tiempo trabajando en la empresa, número de empleos fuera de la empresa en los últimos cinco años, tiempo trabajando en el puesto y número de puestos dentro de la empresa. 
cuatro de las cinco medidas empleadas por Srole (Roberts y Rokeach, 1956) para medir la anomia. De éstas, tres formaron una escala conformada de la siguiente manera: si es mejor aprovechar lo que nos brinda la vida o esperar el futuro; acuerdo o desacuerdo con la declaración de que uno no sabe en quién confiar; y su opinión general acerca de la mayoria de la gente. Se midió el stress mental empleando una escala desarrollada por Leighton y utilizada con éxito en Canadá (Leighton, 1959; Hughs, et al., 1960; y Leighton, et al., 1963a) y en Nigeria (Leighton, et al., 1963b). Una versión reducida se usó con éxito en Puerto Rico (Rogler y Hollingshead, 1965), y en el Perú (Kellert, et al., 1967). La versión original también se ha empleado con éxito en el Perú (Valdivia Ponce, $1970 ; 1972$ ). La escala utilizada en el presente estudio es la utilizada por Kellert y sus asociados, con algunas pequeñas modificaciones. ${ }^{4}$

\section{Metodología de ANÁlisis}

Hay varios estudios (Martínez, 1968; 1972; Bértoli y Portocarrero, 1968; y Muñoz y de Oliveira, 1972; por ejemplo) que demuestran las diferencias entre la población migrante y la población nativa o no migrante, en términos de sexo, edad y estado marital. Valdivia Ponce (1970) ha demostrado que la clase social es una variable importante que distingue nativos e inmigrantes; y otros estudios nuestros (Scurrah y Montalvo, 1975a; 1975b) indican la influencia de esta variable en variables dependientes tales como las actitudes y orientaciones y las medidas de adaptación psicológica. Pensamos también que las diferentes unidades organizacionales donde trabajaban los encuestados y su grupo étnico, podrían ser factores fluyentes. Por eso y porque queríamos concentrarnos en el análisis de la influencia del proceso de inmigración a la ciudad en las variables dependientes, decidimos operar en todos los análisis hechos, con base en el control de los efectos de las variables arriba mencionadas.

El procedimiento utilizado en el estudio fue el del análisis de regresión. Se pueden controlar los efectos de terceras variables si se utilizan tabulaciones cruzadas, pero cuando se trata de controlar más de dos variables, la presentación de cuadros se hace demasiado complicada. El mismo resultado puede lograrse por medio de un análisis de regresión en el cual se incluyen las variables controladas con la variable o variables. independientes, si se mide por medio de la estadística beta (coeficiente de regresión parcial o estandarizada), la contribución independiente de cada variable independiente o de control o la explicación de la variante en la variable dependiente. De esta manera se puede medir la influencia

4 La escala empleada buscaba evidencia de los siguientes síntomas psicosomáticos: salud en general, incomodidad física, problemas estomacales, cansancio, dificultad en reconciliar el sueño, sabor amargo en la boca, pesadillas, sudores fríos, dolores de cabeza, falta de apetito, dolores en el cuerpo, temblor de manos y debilidad general. 
de la variable independiente en el comportamiento de la variable dependiente controlada por las influencias de otras variables. Estas ventajas nos condujeron al empleo del mencionado tipo de análisis.

Por tradición se ha considerado que el análisis de regresión puede emplearse solamente para el análisis de aquellos datos que reúnen las características estrictas de una distribución normal y la cardinalidad. Sin embargo, en los últimos años, varios estudios metodológicos (Boyle, 1966; 1970; Labonitz, 1970) indican que se pueden relajar estos requerimientos, especialmente el que se refiere a la cardinalidad, sin distorsionar los resultados obtenidos.

\section{Resultados}

\section{Migración interna y actitudes y orientaciones}

Primero se examina la relación entre la experiencia migratoria y las actitudes y orientaciones de los trabajadores encuestados. En el cuadro 1 se presentan estas relaciones en cuanto a los ambientes urbanos y rurales. Cuando consideramos el lugar de nacimiento, encontramos que los trabajadores nacidos en un ambiente urbano son menos fatalistas, en general son más confiados y laboran en grupos de trabajo que se caracterizan por su alto grado de solidaridad. En cuanto al lugar de socialización (hasta los 15 años) el patrón resulta muy parecido, salvo que los trabajadores socializados en un ambiente urbano son también menos fatalistas en cuanto a la utilidad de trabajar más y también con respecto a la suerte.

El lugar donde la persona pasó la mayor parte de su vida no está relacionado de la misma manera en sus actitudes y orientaciones. Mientras que quienes han pasado la mayor parte de su vida en un ambiente urbano son menos fatalistas con respecto al valor de trabajar más, lo son más en cuanto a la utilidad de tener una buena preparación personal. No hay diferencia entre quienes han pasado dicho período en un ambiente rural en cuanto a su nivel de confianza, pero sí en términos de solidaridad de su grupo de trabajo, tal como ocurre con las variables anteriores. Los trabajadores que han pasado la mayor parte de su vida en un ambiente urbano valoran más el conformismo y menos la independencia que quienes han pasado la mayor parte de su vida en un ambiente rural.

Se han resumido las influencias del lugar de nacimiento, del lugar de socialización y del lugar donde se pasó la mayor parte de su vida, en una escala total que refleja la influencia del ambiente rural versus el ambiente urbano. Los resultados nos indican que, en contraste con el ambiente rural, el ambiente urbano se asocia con menos fatalismo respecto al valor de trabajar y a la influencia de la suerte; con más confianza; y con más solidaridad entre los compañeros de trabajo. Vale la pena enfatizar que no hay diferencia significativa entre los dos ambientes 
Cuadro 1

RELACIÓN ENTRE LOS AMBIENTES URBANOS Y RURALES Y LAS ACTITUdES $Y$ ORIENTACIONES DE TRABAJADORES DE EMPRESAS COMERCIALES PERUANAS ${ }^{a}$

\begin{tabular}{|c|c|c|c|c|}
\hline Aotitudas y orientaciones & $\begin{array}{l}\text { Lugar do } \\
\text { naoimion to }\end{array}$ & $\begin{array}{l}\text { Lugar de so- } \\
\text { oialtzación }\end{array}$ & $\begin{array}{l}\text { Lugar donde pa- } \\
\text { so la mayor par } \\
\text { te de la vida }\end{array}$ & $\begin{array}{l}\text { Bscala total } \\
\text { rural-urbano }\end{array}$ \\
\hline \multirow{3}{*}{$\begin{array}{l}\text { Optimiamo - escala total } \\
\text { optimismo on oomparación con } \\
\text { el paaado } \\
\text { optimismo acorca del futuro } \\
\text { optimismo aceroa del pais. } \\
\text { Optimiamo acerca de au aituzoión } \\
\text { personal }\end{array}$} & 0.001 & -0.023 & 0.007 & -0.008 \\
\hline & $\begin{array}{r}0.007 \\
-0.019 \\
0.026\end{array}$ & $\begin{array}{l}-0.012 \\
-0.026 \\
-0.010\end{array}$ & $\begin{array}{r}0.027 \\
-0.029 \\
-0.037\end{array}$ & $\begin{array}{r}0.010 \\
-0.034 \\
-0.006\end{array}$ \\
\hline & -0.029 & -0.027 & 0.054 & -0.006 \\
\hline Fatalismo - oscala total & $0.056^{\mathrm{b}}$ & $0.067^{\mathrm{b}}$ & -0.017 & 0.050 \\
\hline $\begin{array}{l}\text { Fatalismo aoerca do la utilidad } \\
\text { do trabajar }\end{array}$ & 0.057 & $0.094^{b /}$ & $0.071^{b /}$ & $0.090^{b}$ \\
\hline $\begin{array}{l}\text { porsonel } \\
\text { Patalisma aoeroa de la suerte }\end{array}$ & $\begin{array}{l}0.006 \\
0.042\end{array}$ & $-0.005 \mathrm{~b}$ & $-0.057^{\circ}$ & $-0.017 \%$ \\
\hline $\begin{array}{l}\text { Confianza - oscala brove } \\
\text { Confianza - esoala larga }\end{array}$ & $0.009 \mathrm{~b}$ & $-0.014 \mathrm{~b}$ & $\begin{array}{r}-0.004 \\
0.004\end{array}$ & $\begin{array}{l}0.001 \mathrm{~b} \\
0.096 \mathrm{~b}\end{array}$ \\
\hline $\begin{array}{l}\text { Alienaoion - escala total } \\
\text { Alienaoión dol puesto } \\
\text { Alienacion de la empresa } \\
\text { Alienaoion del grupo do trabajo }\end{array}$ & $\begin{array}{l}0.041 \\
0.034 \\
0.015 \\
0.043\end{array}$ & $\begin{array}{r}-0.006 \\
0.005 \\
-0.028 \\
0.021\end{array}$ & $\begin{array}{l}0.020 \\
0.047 \\
0.018 \\
0.042\end{array}$ & $\begin{array}{l}0.029 \\
0.037 \\
0.006 \\
0.046\end{array}$ \\
\hline $\begin{array}{l}\text { Solidaridad del grupo de trabajo } \\
\text { Independenoia yeraug conformiamo } \\
\text { Autoritariamo } \\
\text { Oxientación temporal }\end{array}$ & $\begin{array}{l}0.108^{b} \\
0.037 \\
0.039 \\
-0.020\end{array}$ & $\begin{array}{l}0.070^{b} \\
-0.005 \\
-0.028 \\
-0.049\end{array}$ & $\begin{aligned} 0.111 \mathrm{~b} / \\
-0.090 \\
-0.031 \\
0.011\end{aligned}$ & $\begin{array}{l}0.116^{b} \\
-0.018 \\
-0.004 \\
-0.026\end{array}$ \\
\hline
\end{tabular}

a Las cifras representan coeficientes de regresión estandarizados. Cada variable independiente ha sido controlada por los efectos de la empresa donde trabaja el entrevistado, su sexo, su edad, su estado civil, su grupo étnico y su clase social.

b $\mathrm{p} \leq 0.01$.

c $\mathrm{p} \leq 0.05$

en cuanto a optimismo, alienación, valoración de independencia versus conformidad, autoritarismo y orientación temporal.

Otra manera de examinar el contraste cultural asociado con el proceso migratorio, es la de utilizar como variable el área de origen, empleando como polos opuestos Lima-Callao por un lado y la Sierra por el otro. El cuadro 2 indica que los trabajadores nacidos en Lima Metropolitana son menos optimistas en general y específicamente acerca del futuro y de su situación personal pero que en ninguna otra variable hay una diferencia estadísticamente significativa. Cuando analizamos la variable ecológico-cultural por medio del lugar de socialización, resulta asimismo que los trabajadores socializados en Lima-Callao son menos optimistas acerca del futuro y de su situación personal, mientras que los socializados en la Sierra son más optimistas. Sin embargo, los trabajadores socializados en Lima-Callao son menos fatalistas acerca de la necesidad de trabajar y más confiados que los trabajadores socializados en la Sierra. 
No hay diferencia alguna, en términos de optimismo, entre los trabajadores que han pasado la mayor parte de su vida en diferentes zonas pero sí se advierte que los de Lima son menos fatalistas acerca del valor de trabajar más pero más fatalistas en cuanto a la utilidad de una buena preparación personal. Los trabajadores que han pasado la mayor parte de su vida en Lima trabajan en grupos más solidarios y valoran más el conformismo y menos la independencia que los trabajadores que han pasado la mayor parte de su vida en la Sierra.

Cuadro 2

LA RELACIÓN ENTRE EL ÁREA DE ORIGEN Y LAS ACTITUDES Y ORIENTACIONES DE TRABAJADORES EN EMPRESAS COMERCIALES PERUANAS

\begin{tabular}{|c|c|c|c|c|}
\hline Actitudes y orlentaciones & $\begin{array}{l}\text { Lugar do } \\
\text { naoimiento }\end{array}$ & $\begin{array}{l}\text { Lugar do so- } \\
\text { olalizaoion }\end{array}$ & $\begin{array}{l}\text { Luger donde pa- } \\
\text { so la mayor par } \\
\text { te de la vida }\end{array}$ & $\begin{array}{l}\text { Esoala total } \\
\text { rural-urbano }\end{array}$ \\
\hline \multirow{3}{*}{$\begin{array}{l}\text { Optimiamo - oscala total } \\
\text { Optimiamo en comparación con } \\
\text { ol pasado } \\
\text { Optimiamo aoeroa del futuro } \\
\text { optimismo aoroa del pals } \\
\text { Optimiamo acoroa do su situacion } \\
\text { personal }\end{array}$} & $-0.085^{8}$ & -0.042 & -0.002 & -0.057 \\
\hline & $\begin{array}{l}-0.042 g \\
-0.121^{g} \\
-0.047\end{array}$ & $\begin{array}{l}-0.019 b \\
-0.063 \mathrm{~b} \\
-0.006\end{array}$ & $\begin{array}{r}0.030 \\
-0.052 \\
-0.038\end{array}$ & $\begin{array}{l}-0.0139 \\
-0.103^{9} \\
-0.034\end{array}$ \\
\hline & $-0.101^{\mathrm{a} /}$ & $-0.068^{\mathrm{g}}$ & 0.038 & $-0.065^{b / 2}$ \\
\hline \multirow{3}{*}{$\begin{array}{l}\text { Fataliamo - osoala total } \\
\text { Fataliamo aooroa do la utilidad } \\
\text { de trabsjar } \\
\text { Fatmliamo acoroa de la preparaoión } \\
\text { personal } \\
\text { Fatalismo acerea de la suerto } \\
\text { Patalismo aceroa do la "vara" }\end{array}$} & 0.009 & 0.021 & -0.041 & -0.003 \\
\hline & 0.029 & $0.061^{b /}$ & $0.059^{b /}$ & $0.060^{\mathrm{b} /}$ \\
\hline & $\begin{array}{r}-0.029 \\
0.016 \\
-0.014\end{array}$ & $\begin{array}{r}-0.053 \\
0.036 \\
-0.022\end{array}$ & $\begin{array}{l}-0.080^{2} \\
0.002 \\
-0.011\end{array}$ & $\begin{array}{l}-0.062^{b /} \\
0.024 \\
-0.019\end{array}$ \\
\hline $\begin{array}{l}\text { Contianza - esoala breve } \\
\text { Confianza - esoala larga }\end{array}$ & $\begin{array}{r}-0.029 \\
0.045\end{array}$ & $\begin{array}{r}-0.014 \\
0.071^{\mathrm{g}}\end{array}$ & $\underset{0.042}{-0.041}$ & $\begin{array}{r}-0.027 \\
0.044\end{array}$ \\
\hline $\begin{array}{l}\text { Allenooibn - esoala total } \\
\text { Alionaoibn del puosto } \\
\text { Alienaoibn de la ompresa } \\
\text { Llienaoibn del grupo do trabajo }\end{array}$ & $\begin{array}{r}0.003 \\
0.021 \\
-0.027 \\
0.028\end{array}$ & $\begin{array}{r}-0.029 \\
0.001 \\
-0.032 \\
-0.010\end{array}$ & $\begin{array}{r}-0.007 \\
0.034 \\
0.002 \\
0.027\end{array}$ & $\begin{array}{r}-0.007 \\
0.025 \\
-0.021 \\
0.025\end{array}$ \\
\hline $\begin{array}{l}\text { Solidaridad del grupo de trabajo } \\
\text { Independenola yergue conforminmo } \\
\text { Autoritari amo } \\
\text { oriontaoion temporal }\end{array}$ & $\begin{array}{r}0.020 \\
-0.020 \\
0.012 \\
-0.005\end{array}$ & $\begin{array}{r}0.044 \\
-0.014 \\
-0.003 \\
-0.045\end{array}$ & $\begin{array}{l}0.076^{\mathrm{g}} \\
-0.125^{9} \\
-0.055 \\
0.010\end{array}$ & $\begin{array}{l}0.056 \\
-0.058 \\
-0.009 \\
-0.018\end{array}$ \\
\hline
\end{tabular}

$\mathrm{a} \mathrm{p} \leq 0.01$.

b $\mathrm{p} \leq 0.05$.

Al resumir estas tres variables en una escala total que refleja la influencia ecológico-cultural, resulta que Lima se asocia con un pesimismo relativo acerca del futuro y de la situación personal del trabajador; con menos fatalismo respecto a la utilidad de trabajar duro; y con un fatalismo relativo en cuanto a la utilidad de una buena preparación personal. No hay diferencias entre las zonas ecológico-culturales en cuanto a confianza, alienación, solidaridad del grupo de trabajo, valoración de independencia versus conformismo, autoritarismo y orientación temporal.

En el cuadro 3 tratamos de resumir la influencia del ambiente urba no sobre las actitudes y orientaciones de los trabajadores por medio de una variable que mide su grado de exposición a Lima-Callao. Los traba- 
jadores con más exposición a la ciudad son más pesimistas acerca de su situación personal y menos fatalistas acerca de la utilidad de trabajar duro y de la influencia de la suerte, y son más confiados. Otra manera de resumir las influencias relativas de la ciudad y el campo, fue la de establecer una escala incluyendo las dos medidas del lugar de nacimiento, las dos medidas del lugar de socialización y las dos medidas del lugar donde se pasó la mayor parte de la vida. La única diferencia entre la escala total y la escala parcial es que la primera también incluye la medida de la exposición a Lima-Callao. Estas dos escalas nos señalan que la ciudad está asociada con más pesimismo acerca del futuro, menos fatalismo acerca de la utilidad de trabajar duro, más confianza y más solidaridad en su grupo de trabajo.

\section{Cuadro 3}

LA RELACIÓN ENTRE MIGRACIÓN Y EXPOSICIÓN A LIMA Y LAS ACTITUDES $Y$ ORIENTACIONES DE TRABAJADORES EN EMPRESAS COMERCIALES PERUANAS

\begin{tabular}{|c|c|c|c|}
\hline Actitudes $y$ orientaoiones & Bxposioisn a Lima & $\begin{array}{l}\text { Bacala Paroial } \\
\text { Migraoión Interna }\end{array}$ & $\begin{array}{c}\text { Eacala Total Mrgracion } \\
\text { Interna }\end{array}$ \\
\hline \multirow{3}{*}{ 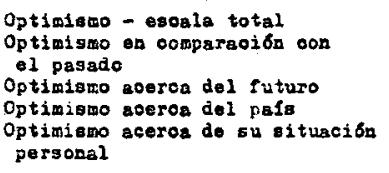 } & -0.053 & -0.036 & -0.041 \\
\hline & $\begin{array}{l}-0.049 \\
=0.038 \\
-0.023\end{array}$ & $\begin{array}{l}-0.002 \\
-0.076^{g} \\
-0.022\end{array}$ & $\begin{array}{l}-0.016 \\
-0.063^{b} \\
-0.024\end{array}$ \\
\hline & $-0.068^{2 / 2}$ & -0.039 & -0.048 \\
\hline \multirow{3}{*}{$\begin{array}{l}\text { Patalismo - oscala total } \\
\text { Fatalismo aoeroa de la utilidad } \\
\text { da traba jar } \\
\text { Pataliamo aoerca de preparación } \\
\text { pergonal } \\
\text { Fatalismo acerca de la suerto } \\
\text { Fatalismo acerca de la "vara" }\end{array}$} & 0.040 & 0.024 & 0.022 \\
\hline & $0.083^{\mathrm{g} /}$ & $0.080^{2 /}$ & $0.075^{2 /}$ \\
\hline & $\begin{array}{l}-0.019 \mathrm{~g} \\
0.06 \mathrm{~g}^{\mathrm{g}} \\
-0.000\end{array}$ & $\begin{array}{r}-0.044 \\
0.044 \\
-0.002\end{array}$ & $\begin{array}{r}-0.037 \\
0.053 \\
-0.012\end{array}$ \\
\hline $\begin{array}{l}\text { Confianza - eacala breve } \\
\text { Coafianza - esoala larga }\end{array}$ & $\begin{array}{l}0.004 \\
0.072^{2 /}\end{array}$ & -0.014 & $-0.005 \mathrm{~s}$ \\
\hline 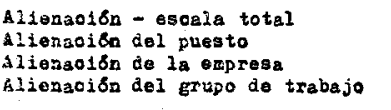 & $\begin{array}{r}0.010 \\
0.026 \\
-0.011 \\
0.006\end{array}$ & $\begin{array}{r}0.012 \\
0.033 \\
-0.008 \\
0.039\end{array}$ & $\begin{array}{r}0.001 \\
0.015 \\
-0.008 \\
0.021\end{array}$ \\
\hline $\begin{array}{l}\text { Solidaridad del grupo do trabajo } \\
\text { Independenota vezsus oonformiswo } \\
\text { Autoriterismo } \\
\text { Orientaoion temperal }\end{array}$ & $\begin{array}{r}0.056 \\
-0.005 \\
0.028 \\
-0.047\end{array}$ & $\begin{array}{l}0.091^{\mathrm{g}} \\
-0.041 \\
-0.006 \\
-0.023\end{array}$ & $\begin{array}{r}0.083^{3 / 2} \\
-0.030 \\
0.005 \\
-0.035\end{array}$ \\
\hline
\end{tabular}

$\mathrm{a} \mathrm{p} \leq 0.01$.

o $\mathrm{p} \leq 0.05$.

\section{Migración interna, aspiraciones y movilidad}

En el cuadro 4 se presentan los resultados de la relación entre la dimensión rural-urbana y las medidas de aspiraciones y movilidad. Resulta que los trabajadores nacidos en el ambiente urbano aspiran a ocupaciones de estatus relativamente más bajo para sus hijos que los trabajadores nacidos en el ambiente rural, pero, en contraste, están más motivados por 
la aspiración a mejorar. Este último hallazgo se repite en términos del lugar de socialización, pero resulta que los trabajadores socializados en el campo han logrado una mayor movilidad social que los socializados en Lima-Callao. Los trabajadores que han pasado la mayor parte de su vida en la ciudad se encuentran más satisfechos con su trabajo pero también han experimentado menos ascenso social. Resumiendo lo referente a la influencia relativa del ambiente rural versus la del ambiente urbano, encontramos que el ambiente urbano se asocia con un mayor deseo de mejorar pero con menor movilidad social hacia arriba que el ambiente rural.

\section{Cuadro 4}

RELACIÓN ENTRE LOS AMBIENTES URBANOS Y RURALES Y LAS ASPIRACIONES $Y$ LA MOVILIDAD DE LOS TRABAJADORES DE EMPRESAS COMERCIALES PERUANAS ${ }^{a}$

\begin{tabular}{|c|c|c|c|c|}
\hline Variables dependientes & $\begin{array}{l}\text { Lugar de } \\
\text { naoimiento }\end{array}$ & $\begin{array}{l}\text { Lugar do so- } \\
\text { oial12a016n }\end{array}$ & $\begin{array}{l}\text { Lugar donde pa- } \\
\text { of la mayor paz } \\
\text { to de la vida }\end{array}$ & $\begin{array}{l}\text { Eaoala total } \\
\text { rural-urbano }\end{array}$ \\
\hline $\begin{array}{l}\text { Aapiraoionea aloanzadad } \\
\text { Aspiraoiones para l futuro } \\
\text { Satiafaceisn } \\
\text { Deseo do mejorar }\end{array}$ & $\begin{array}{l}-0.018 \\
-0.066^{\mathrm{b}} \\
0.001 \\
0.075^{\circ}\end{array}$ & $\begin{array}{l}-0.018 \\
-0.021 \\
-0.007 \\
0.075\end{array}$ & $\begin{array}{l}0.016 \\
-0.035 \\
0.070 \% \\
0.016\end{array}$ & $\begin{array}{l}-0.012 \\
-0.049 \\
0.018 \\
0.073^{9}\end{array}$ \\
\hline $\begin{array}{l}\text { Kovilidad sooial } \\
\text { Kovilldad ooupaoional }\end{array}$ & $\begin{array}{r}-0.051 \\
0.034\end{array}$ & $\begin{array}{l}-0.074^{9} \\
0.030^{\prime}\end{array}$ & $\begin{array}{l}-0.089^{0} \\
0.004\end{array}$ & $\begin{array}{l}-0.0810 / \\
0.034\end{array}$ \\
\hline
\end{tabular}

a Las cifras representan coeficientes de regresión estandarizados. Cada variable independiente ha sido controlada por los efectos de la empresa donde trabajaba el entrevistado, su sexo, edad, estado civil, grupo étnico y clase social.

b $\mathrm{p} \leq 0.05$.

c $\mathrm{p} \leq 0.01$.

Como indican las cifras del cuadro 5 , el lugar de nacimiento definido en términos de la región ecológico-cultural, no tienen ninguna relación significativa con las medidas de aspiraciones y movilidad. El lugar de socialización está relacionado negativamente con la movilidad social, indicándonos que los trabajadores socializados en Lima-Callao han experimentado menor movilidad social hacia arriba, que los trabajadores socializados en la Sierra. Los trabajadores que han pasado la mayor parte de su vida en Lima-Callao están más satisfechos con su trabajo y también han experimentado menor movilidad social hacia arriba que quienes han pasado la mayor parte de su vida fuera de Lima, especialmente en la Sierra. Resumiendo lo relativo a la influencia de la zona ecológicocultural, encontramos que solamente la movilidad social está asociada con esta variable.

El grado de exposición a Lima-Callao no está asociado significativamente con ninguna medida de aspiraciones o movilidad, como lo indican los resultados del cuadro 6. La escala parcial de migración interna revela que el ambiente metropolitano induce un mayor deseo de mejorar pero presenta al trabajador con una menor posibilidad de ascenso social que 
Cuadro 5

RELACIÓN ENTRE EL ÁREA DE ORIGEN Y LAS ASPIRACIONES Y LA MOVILIDAD DE LOS TRABAJADORES DE EMPRESAS COMERCIALES PERUANAS

\begin{tabular}{|c|c|c|c|c|}
\hline Variables dependientes & $\begin{array}{l}\text { Lugar do } \\
\text { naoimien to }\end{array}$ & $\begin{array}{l}\text { Lugar de so- } \\
\text { oial1saoton }\end{array}$ & $\begin{array}{l}\text { Lugar donde pa- } \\
\text { so la mayor par } \\
\text { to de la vida }\end{array}$ & $\begin{array}{l}\text { Escala total } \\
\text { Grea do origen }\end{array}$ \\
\hline $\begin{array}{l}\text { Aspiraotonea aloansadas } \\
\text { Aspiraciones para ol futura } \\
\text { Satisfaodisa } \\
\text { Deseo de mejorar } \\
\text { Movilidad soofal } \\
\text { Movilidad ooupacionsl }\end{array}$ & $\begin{array}{r}-0.032 \\
-0.056 \\
0.017 \\
0.052 \\
-0.029 \\
0.016\end{array}$ & $\begin{array}{l}-0.058 \\
0.001 \\
0.018 \\
0.031 \\
-0.072^{8} \\
0.024\end{array}$ & $\begin{array}{l}0.032 \\
-0.035 \\
0.080^{8} \\
-0.013 \\
-0.085 \mathrm{~g} \\
0.006\end{array}$ & $\begin{array}{l}-0.028 \\
-0.032 \\
0.047 \\
0.034 \\
-0.070^{8} \\
0.022\end{array}$ \\
\hline
\end{tabular}

a $\mathrm{p} \leq 0.01$.

en el ambiente serrano-rural. Asimismo, si se toma en consideración la exposición a la ciudad de Lima, la escala total confirma el resultado en términos de la movilidad social y además indica una mayor movilidad ocupacional en el ambiente urbano.

\section{Cuadro 6}

RELACIÓN ENTRE MIGRACIÓN Y EXPOSICIÓN A LIMA Y LAS ASPIRACIONES Y LA MOVILIDAD DE LOS TRABAJADORES DE EMPRESAS COMERCIALES DE LIMA

\begin{tabular}{|c|c|c|c|}
\hline Variables dependiantes & Exposición a Lima & $\begin{array}{l}\text { Esoala parotial } \\
\text { migración interna }\end{array}$ & $\begin{array}{c}\text { Egoal a Total } \\
\text { migración interna }\end{array}$ \\
\hline $\begin{array}{l}\text { Aspiracionea aloanzadas } \\
\text { Aspiraoionea para l futuro } \\
\text { Satisfaocibn } \\
\text { Deseo do me jorar } \\
\text { Movilidad goolal } \\
\text { Movilidad ocupacional }\end{array}$ & $\begin{array}{r}-0.031 \\
-0.042 \\
0.037 \\
0.040 \\
-0.041 \\
0.039\end{array}$ & $\begin{array}{l}-0.022 \\
-0.044 \\
0.035 \\
0.058 \mathrm{~g} \\
-0.081 \\
0.029\end{array}$ & $\begin{array}{l}-0.032 \\
-0.053 \\
0.031 \\
0.060 \\
-0.069 \mathrm{~b} / \\
0.030 \mathrm{~b}\end{array}$ \\
\hline
\end{tabular}

a $\mathrm{p} \leq 0.05$.

${ }^{\mathrm{b}} \mathrm{p} \leq 0.01$.

\section{3) Migración interna y adaptación psicológica}

Valdivia Ponce (1970) ha encontrado un nivel relativamente alto de desadaptación psicológica entre los inmigrantes en Lima comparando con los limeños nativos. El cuadro 7 confirma con evidencia esos hallazgos. Los trabajadores nacidos en una zona rural experimentan más anomia y stress mental de los trabajadores nacidos en Lima-Callao. También experimentan mayor stress mental los trabajadores socializados en el campo o quienes han pasado la mayor parte de su vida en el campo. En general, el ambiente rural se asocia con más anomia y mayor stress mental, que el ambiente urbano. Cuando examinamos la influencia de la zona ecológico-cultural, encontramos que los trabajadores nacidos fuera de Lima, socializados fuera de Lima o que han pasado la mayor parte de su 
vida fuera de ella, sufren de mayor stress mental que los limeños. Finalmente, un patrón similar emerge de una consideración del grado de exposición a Lima Metropolitana. A mayor grado de exposición, menos anomia y menor stress mental.

\section{Cuadro 7}

RELACIONES ENTRE EL AMBIENTE, ÁREA DE ORIGEN Y EXPOSICIÓN A LIMA Y EL GRADO DE ANOMIA Y "STRESS" MENTAL EN TRABAJADORES DE EMPRESAS COMERCIALES PERUANAS a

\begin{tabular}{|c|c|c|}
\hline Variables independientes & Anoria & "Streas" mental" \\
\hline \multicolumn{3}{|l|}{ Dimension rural-urbana: } \\
\hline $\begin{array}{l}\text { Lugar de naoimiento } \\
\text { Lugar de socializaci6n } \\
\text { Lugar donde paso la mayor parte de la vida } \\
\text { Eacala total: rural-urbana }\end{array}$ & $\begin{array}{l}0.106^{\mathrm{b}} \\
0.056 \\
-0.007 \mathrm{~b} \\
0.068^{\mathrm{b}}\end{array}$ & $\begin{array}{l}0.117 \\
0.131 \\
0.066 \\
0.135\end{array}$ \\
\hline \multicolumn{3}{|l|}{ Dimengibn zone ocológiao-oultural: } \\
\hline $\begin{array}{l}\text { Lugar de nacimiento } \\
\text { Lugar de soolalizacion } \\
\text { Lugar donde pas6 la rrayor parte de la vida } \\
\text { Becala total : area de origen }\end{array}$ & $\begin{array}{r}0.026 \\
0.026 \\
-0.031 \\
0.012\end{array}$ & $\begin{array}{l}0.143 \\
0.127 \\
0.067 \\
0.146\end{array}$ \\
\hline \multicolumn{3}{|l|}{ Besumen } \\
\hline $\begin{array}{l}\text { Exposiaion a Liwa } \\
\text { Bgcala paroial - migracion interna } \\
\text { Bgoala total - migracion interna }\end{array}$ & $\begin{array}{l}0.062^{\circ} \\
0.042 \\
0.046\end{array}$ & $\begin{array}{l}0.114 \\
0.151 \\
0.141\end{array}$ \\
\hline
\end{tabular}

a Las cifras representan coeficientes de regresión estandarizados. Cada variable independiente ha sido controlada por los efectos de la empresa donde trabaja el entrevistado, su sexo, edad, estado civil, grupo étnico y clase social.

b $\mathrm{p} \leq 0.01$.

c $\mathrm{p} \leq 0.05$.

\section{RESUMEN Y CONCLUSIONES}

¿Qué nos dicen estos resultados acerca de las diferencias entre los trabajadores formados en el campo y los formados en la metrópoli y respecto al proceso de migración en general?

Primero, que hay menos diferencias de las que uno esperaría en términos de actitudes y orientaciones. Por ejemplo, los problemas de adaptación al ambiente urbano experimentados por el trabajador inmigrante no resultan en un mayor grado de alienación y la mayor exposición al ambiente urbano del trabajador limeño tampoco aumenta su alienación. Al contrario de lo que se podría esperar, el trabajador urbano no exhibe una orientación mayor hacia el futuro que el trabajador formado en el más tradicional sector rural. Por último, el autoritarismo, una cualidad de la personalidad, no difiere entre el trabajador de la ciudad y el del campo.

En general, el trabajador urbano es menos optimista que quien viene del campo o de la Sierra o quien tiene menos exposición a Lima, espe- 
cialmente respecto a su situación personal y sus esperanzas para el futuro. Este hallazgo encuentra apoyo en las conclusiones de las investigaciones de Mangin (1967) en las barriadas de Lima. El trabajador limeño es menos fatalista respecto del valor de trabajar más y de la influencia de la suerte pero más fatalista acerca de la ventaja que representa una buena preparación personal. El mayor grado de creencia en la suerte por parte del trabajador inmigrante puede reflejar su falta de experiencia en la ciudad, y su mayor fe en la preparación personal encuentra un paralelo en el hallazgo, en numerosos estudios, de que la búsqueda de una mejor educación es una de las razones dadas con más frecuencia como motivo para migrar a Lima. El mayor énfasis puesto por los limeños en la necesidad de trabajar duro puede reflejar una cierta desmistificación de la realidad dura de la vida urbana y cierta proletarización. Los trabajadores limeños son más confiados y trabajan en grupos que son más solidarios. No se sabe si trabajan mayormente con otros limeños o con una mezcla de limeños y provincianos. Los limeños también son ligeramente más conformistas.

Los trabajadores inmigrantes han alcanzado ligeramente más sus aspiraciones y tienen mayores aspiraciones para el futuro que los trabajadores limeños pero, quizás debido a estas ambiciones, están menos satisfechos con su trabajo.

El trabajador migrante, entonces, ha experimentado mayor movilidad social y aspira aún más; es optimista y valora su independencia. Pero también es fatalista, salvo en cuanto al valor de una buena preparación personal, y no confía en los demás. En términos económico-sociales ha mejorado más que el trabajador urbano pero sigue adoptando actitudes tradicionales tales como la desconfianza y el fatalismo. El progreso que ha ganado por migrar a la ciudad parece que es a costa de un mayor grado de anomia y un nivel de stress mental marcadamente mayor.

\section{REFERENCIAS BIBLIOGRÁFICAS}

1968. J. Oscar Alers, Richard P. Appelbaum, "La migración en el Perú: Un inventario de proposiciones", Estudio de Población y Desarrollo (1) 4.

1968. Fernando Bértoli y Felipe Portocarrero, "La modernización y la migración interna en el Perú, Lima: Instituto de Estudios Peruanos (serie: urbanización, migraciones y cambios).

1966. Richard P. Boyle, "Causal Theory and Statistical Measures of Effect: A Convergence". American Sociological Review, 31: 843-850.

1970. ㄱ. "Path Analysis and Ordinal Data", American Journal of Sociology, 75. $461-480$.

1968. Harley L. Browning y Waltraut Feindt, "Diferencias entre la población nativa y la migrante en Monterrey". Demografía y Economía, 2: 183-204.

1972. Centro de Estudios de Población y Desarrollo, "Informe Demográfico del Perú, 1970". Lima: Centro de Estudios de Población y Desarrollo.

1960. Charles C. Hughs, Marc-Adelard Tremblay, Robert N. Rapoport y Alexander H. Leighton, People of Cove and Woodlot, Nueva York: Basic Books.

1967. Stephen Kellert, Lawrence K. Williams, William. F. Whyte y Giorgio Alberti, 
"Culture Change and Stress in Rural Perú: A Preliminary Report". Milbank Memorial Fund Quarterly (45) 4: 391-415.

1970. Sanford Labovitz, "The Assignment of Numbers to Rank Order Categories", American Sociological Review, 35: 515-524.

1959. Alexander H. Leighton, My Name is Legion, Nueva York: Basic Books.

1963. Alexander Leighton, et al., Psychiatric Disorders Among the Yoruba, Ithaca (N. Y.): Cornell University Press.

1963. Dorothea C. Leighton, John S. Harding, David B. Macklin, Allister M. Macmillan y Alexander $\mathrm{H}$. Leighton, The Character of Danger, Nueva York: Basic Books.

1967. William Mangin, "Squatter Settlements", Scientific American (217) 4: 21-28.

1968. Héctor Martínez, William Prado y Jorge Quintanilla, "El éxodo rural en el Perú", Lima: Centro de Estudios de Población y Desarrollo (Documento de trabajo).

1972. Humberto Muñoz y Orlandina de Oliveira, "Migraciones internas y desarro1lo: Algunas consideraciones sociológicas". Demografía Y Economía, 6: 248-260.

1972. Oficina Nacional de Estadística y Censos, "Los pueblos jóvenes en el Perú", Boletín de Análisis Demográfico, 13.

1956. Alan H. Roberts y Milton Rokeach, "Anomie, Authoritarianism, and Prejudice: A Replication", American Journal of Sociology, 61: 355-358.

1965. Lloyd Rogler y August B. Hollingshead, Trapped: Families and Schizophrenia, Nueva York: John Wiley and Sons.

1975. Martin J. Scurrah y Abner Montalvo, "Social Bases of Support for Peru's Revolutionary Military Government" (mimeo), Lima: ESAN.

1975. — "Social Class, Working Conditions and Attitudes and Orientations in Peru", (mimeo), Lima: ESAN.

1970. Óscar Valdivia Ponce, Migración interna a la metrópoli: Contraste cultural, conflicto y desadaptación, Lima: Universidad Nacional Mayor de San Marcos.

1972. —— "El impacto de algunos factores sociales determinantes de patología mental", Acta Psiquiátrica Psicológica de América Latina, 18: 143-153. 